\title{
Relationship between financial well-being and Burnout Syndrome in anesthesiologists
}

\begin{abstract}
Summary
Burnout syndrome and problems in personal financial life can have negative effects on mental health. Given this similarity between the two, the question is whether there is a correlation between financial well-being and the risk of developing the syndrome. In this study a questionnaire was applied to analyze this relationship. The results show that the lower the financial well-being, the higher the risk of Burnout. The prevalence of the syndrome in the sample was $28.6 \%$. Furthermore, age and time in the job market showed a negative correlation with this risk, while the weekly work load showed a positive correlation. Despite not demonstrating causality between the variables, this study presents new data in the literature. Future studiesin the area are necessary to aid in the prevention and treatment of Burnout Syndrome.
\end{abstract}

Keywords: Burnout Syndrome, Financial Well-Being,Anesthesiologists

\author{
Volume I3 Issue 4 - 202 I
}

\author{
Dr. Eduardo Schuch Reimann, Dr.Airton \\ Bagatini \\ Sane Education and Training Center, Ernesto Dornelles Hospital, \\ Brazil
}

Correspondence: Dr. Eduardo Schuch Reimann Sane Education and Training Center, Ernesto Dornelles Hospital, Porto Alegre, RS, Brazil,Tel +555 | 9997 | 2280 ,

Email Baga2020@gmail.com

Received: June 13,2021 | Published: June 22, 2021

\section{Introduction}

The concept of Burnout Syndrome was created by psychologis Cristina Maslach in the seventies. It is characterized as a type of long lasting stress, linked to work situations, which is highly prevalent among healthcare professionals. ${ }^{1}$ The syndrome is composed of three dimensions of symptoms: emotional exhaustion, depersonalization and reduced personal accomplishment. Currently, there are no consensual criteria to define the diagnosis of Burnout. Some authors consider its presence when one of the dimensions is severely affected. ${ }^{2}$ It is a condition with international relevance and significant prevalence in healthcare professionals, mainly physicians. Data from a Canadian oncology sector show prevalence of a high level of emotional exhaustion in 53\% of the physicians, $37 \%$ of other healthcare professionals and $30 \%$ of the support staff. At national level, a 2007 study indicates that $57 \%$ of the Brazilian physicians manifest some worrisome degree of Burnout Syndrome, 23\% being severe and $10 \%$ extreme. ${ }^{3}$ Similar results were found in countries such as Australia, Mexico, Romania, France and Spain. ${ }^{4}$

The syndrome results from constant and repetitive emotional pressure associated with intense interpersonal involvement for long periods of time in the work environment. ${ }^{5}$ Work overload has been demonstrated as one of its most important antecedents. ${ }^{6}$ In a survey carried out by the Medscape site, the causes most often mentioned by physicians were: too many bureaucratic activities, too many hours worked and not enough pay. ${ }^{7}$ Anesthesiology is a specialty with characteristics that contribute differently to Burnout. ${ }^{8}$ Further, the weekly workload of an anesthesiologist may be extensive, as reported in a study carried out in Recife that found a weekly average of 15.5 shifts worked. ${ }^{9}$

Among the symptoms presented by a person with Emotional Exhaustion are feelings of anger, irritability, impatience, and hopelessness. Physical symptoms such as low energy, chronic fatigue, muscle tension, and headache may be present. The individual with Depersonalization may manifest feelings of alienation in relation to others, as they are viewed negatively and avoid contact in the work environment. Finally, the individual with Reduced Personal Accomplishment has a perception that nothing they do is worthwhile or produces meaningful results. ${ }^{8}$ Besides the deleterious consequences at individual level, Burnout also brings damages at the organizational level. It is associated with negative outcomes such as absenteeism, high personnel turnover and reduced productivity. ${ }^{6}$

Regarding the financial issue, debts can be evaluated in a dichotomous manner, being negative when they occur due to lack of financial control, or positive when planned aiming at ventures and acquisition of productive assets. ${ }^{10}$ When the individual loses control of his/her financial life, he/she may present reduced quality and life expectancy and develop symptoms of emotional stress, anxiety, and depression. ${ }^{11-17}$ One study analyzed 52 papers and showed a direct relationship between the size of a debt and the level of concern and stress of the debtor. ${ }^{15}$ These data demonstrate the impact that finances can have on mental health. Despite this, a World Bank survey points out that only $10.9 \%$ of the Brazilian population has the habit of saving money thinking about their future for when they are old. ${ }^{18}$

Preventing Burnout Syndrome can be difficult. Establishing a limit on working hours, practicing regular physical activities, taking frequent vacations, taking breaks during work and adopting a healthy diet are recommended protective strategies. ${ }^{19,20}$ Considering that the weekly workload of an anesthesiologist can be extensive, the execution of preventive measures can be difficult. ${ }^{8,9}$

Despite the vast literature on the prevalence, prevention, and consequences of Burnout Syndrome, little is discussed about the possible reasons that lead professionals to submit themselves to exhaustive workloads. In this sense, it is hypothesized that the desire to obtain greater financial returns is one of these motivators. Meanwhile, works related to financial life are focused on the effects of debt on mental health. However, no studies were found that relate a Burnout score and a financial well-being score. Given the similarities between these variables and the potential synergism of their effects on a professional's mental health, the existence of a correlation between them is questioned. The objective of this study isto analyze the correlation between financial well-being and Burnout Syndrome and itsprevalence among Anesthesiologists working in Rio Grande do Sul. Besides, with thedata collected, we can outline a profile with characteristics of the professional at greater risk of developing the syndrome. 


\section{Materials and methods}

This was a cross-sectional study carried out with the anesthesiologists who work in the state of Rio Grande do Sul and are members of the Anesthesiology Society of Rio Grande do Sul (SARGS). Anesthesiologists with e-mail registered at SARGS received an invitation to participate in the study via mail, and those who agreed were included in the convenience sample of this study. Data was collected from June 29 to July 30, 2020 via an anonymous, self- applied, self-explanatory questionnaire designed on the Google Forms platform containing three sections of questions: personal data, financial well-being, and Burnout Syndrome.

The sociodemographic variables contained in the first section were: age, sex, marital status, years as an anesthesiologist, number of financial dependents, and how they work in the labor market. The analysis of financial well-being used the questionnaire designed and validated by Norvilitis, Szablicki, and Wilson, which is composed of eight questions with answers in a five-point Likert format: I feel uncomfortable with the amount I owe; I worry constantly about paying my debts; I feel financially satisfied; I believe I am ina good financial situation; I think a lot about my debts; I have had arguments with other people (parents, friends, spouse, others) because of my debts; In one yearfrom now I intend to have no debts (financing, loans, credit card, etc.); In five years from now I intend to have no debts (financing, loans, credit card, etc.). ${ }^{20}$

To evaluate the Burnout Syndrome, the Maslach Burnout Inventory (MBI), translated and adapted by Tamayo, was used. ${ }^{21}$ The MBI, considered the standard tool to evaluate the syndrome, contains 22 items, divided into three fundamental domains: Emotional exhaustion, Depersonalization and Professional involvement or accomplishment, with nine, five and eight items, respectively, with responses in Likert format from 0 to 6 points. The score is classified as low, medium, and high risk for developing the syndrome. For the Emotional Exhaustion domain, a score $<14$, medium between 15 and 24, and high, a score $>25$ is considered low. In Depersonalization, score $<3$ means low, between 4 and 9 medium, and $>10$, high. Finally, for professional involvement or fulfillment, scores $>40$ indicate low, between 33 and 39 points is medium, and $<32$, high.

Statistical analysis was performed using SPSS v. 20.0. Categorical variables were described as frequencies and percentages and quantitative variables, according to the Kolmogorov Smirnov test, with symmetrical distribution, were described by the mean and standard deviation and those with asymmetrical distribution, by the median and the interquartile range. Quantitative variables were correlated with each other using the Pearson or Spearman correlation coefficient, according to their distribution. Quantitative variables with normal distribution were compared by Student's $t$ test for independent samples and those with asymmetric distribution by the Mann Whitney test. A $5 \%$ significance level was considered for the established comparisons.

The study was approved by the Research Ethics Committee under protocol CAAE $n^{\circ}$ 30235620.3.0000.5304 and the participants were presented with the Informed Consent Form, via e-mail, prior to completing the questionnaire.

\section{Results}

Among the 1359 anesthesiologists registered in the Anesthesiology Society of Rio Grande do Sul (SARGS), 234 (17.2\%) answered the questionnaire. Mean age was 44.7 years. Male professionals (63.2\%), married $(56.8 \%)$, and working in private anesthesiology teams $(63.7 \%)$ were predominant. The mean weekly workload was 47.6 hours. The median number of years working in the labor market was 14.0, and the median number of financial dependents was 1.0 (the median was used due to theasymmetrical distribution of the data). Other results of the general characteristics of the sample are shown in Table 1. As for the financial score, a mean and standard deviation of $29.5 \pm 6.5$ was found (its score ranges from 8 to 40 points). The internal consistency was acceptable (Cronbach's $\alpha=0.76$ ), while in the original work its value was 0.74 .

Table I General characteristics of the sample

\begin{tabular}{|c|c|}
\hline Age in years* & $44,7 \pm 12,4$ \\
\hline \multicolumn{2}{|l|}{$\operatorname{Sex}(\%)$} \\
\hline Male & 63,2 \\
\hline Female & 36,8 \\
\hline \multicolumn{2}{|l|}{ Marital Status (\%) } \\
\hline Married & 56,8 \\
\hline Single & 18,4 \\
\hline Stable Union & 17,5 \\
\hline Divorced & 5,6 \\
\hline Widow(er) & 0,9 \\
\hline \multicolumn{2}{|l|}{ Main form of action (\%) } \\
\hline Private anesthesiology team & 63,7 \\
\hline Self-employed ("Freelancer") & 15,8 \\
\hline Anesthesiology Resident & 11,5 \\
\hline Work contract with hospitals and/or clinics & 4,7 \\
\hline Civil Servant & 4,3 \\
\hline Weekly workload* & $47,6 \pm 19,9$ \\
\hline Years in the job market\# & $14,0(5,0-25,0)$ \\
\hline Number of financial dependents\# & $\mathrm{I}, 0(0,0-2,0)$ \\
\hline
\end{tabular}

Variables with normal distribution described by the mean \pm standard deviation; Variables with asymmetric distribution described by the median (interquartile range: P25-P75);

$\%$ Categorical variables described by the percentage of $n$

Regarding the Burnout score, the answers were grouped into three categories (low risk, moderate risk, and high risk) according to the risk of a participant developing symptoms of each of the three dimensions. Among the participants, 23.5\% were at high risk for developing symptoms of emotional exhaustion, $9.0 \%$ were at high risk for depersonalization, and $8.5 \%$ were at high risk for reduced personal accomplishment. Thus, $28.6 \%$ of the participants presented high risk in at least one of the dimensions. The prevalence of responses is shown in Table 2 . 
Table 2 Prevalence of risks of developing Burnout symptoms

\begin{tabular}{llll}
\hline & Low risk (\%) & Moderate risk (\%) & High risk (\%) \\
\hline Emotional Exhaustion & 49,1 & 27,4 & 23,5 \\
Depersonalization & 76,9 & 14,1 & 9,0 \\
Reduced personal fulfillment & 73,5 & 17,9 & 8,5 \\
\hline
\end{tabular}

In analyzing the relationship between the score of the financial score and each dimension of the Burnout score we found the result as shown in Figures 1, 2 and 3. The participants who presented low risk in the "Emotional Exhaustion" sphere presented a mean and standard deviation in the financial score of $30.7 \pm 6.0$, while those at high risk presented $26.9 \pm 6.3(\mathrm{p}=0.001)$. After adjusting for age and weekly workload (ANCOVA, analysis of covariance), there continued to be a statistically significant difference in the financial score between participants at low and high risk $(\mathrm{p}<0.001)$. When analyzing the sphere "Depersonalization", the low risk group presenteda financial score of $29.8 \pm 6.3$, while the high risk group presented a score of $28.3 \pm 6.8$ $(p=0.480)$. In the dimension "Reduced personal accomplishment" the low risk group obtained a financial score of $29.9 \pm 6.2$, while the high risk group $28.3 \pm 7.2(\mathrm{p}=0.191)$.

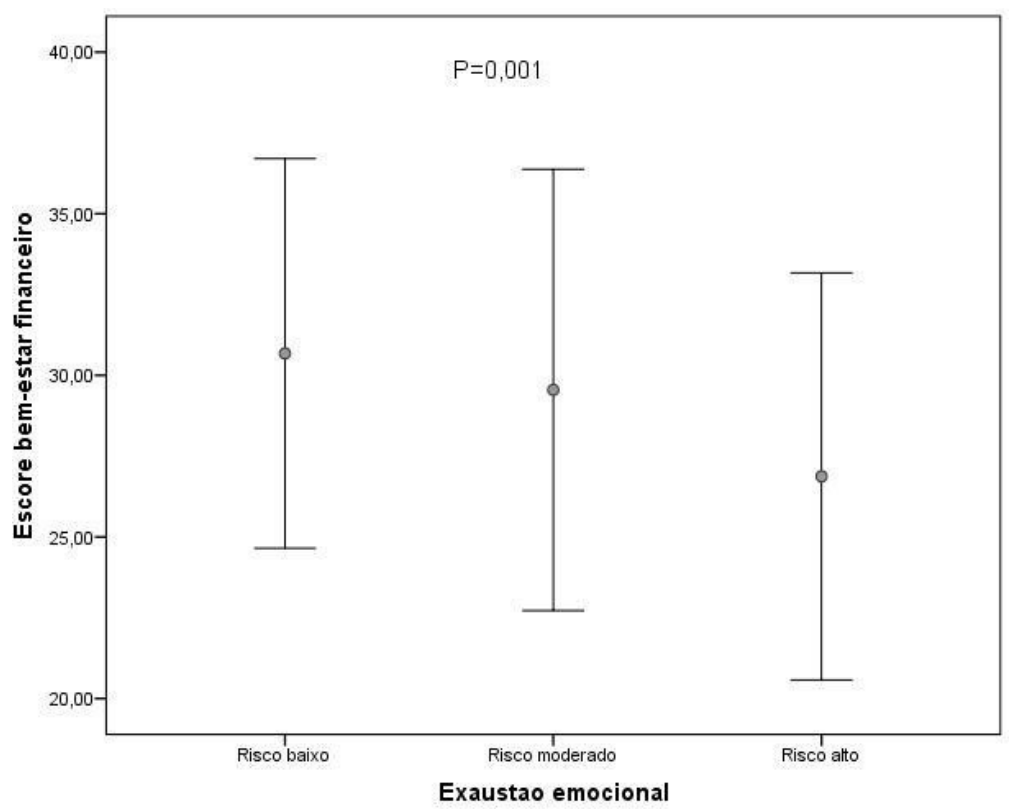

Figure I Relationship between emotional exhaustion and financial well-being.

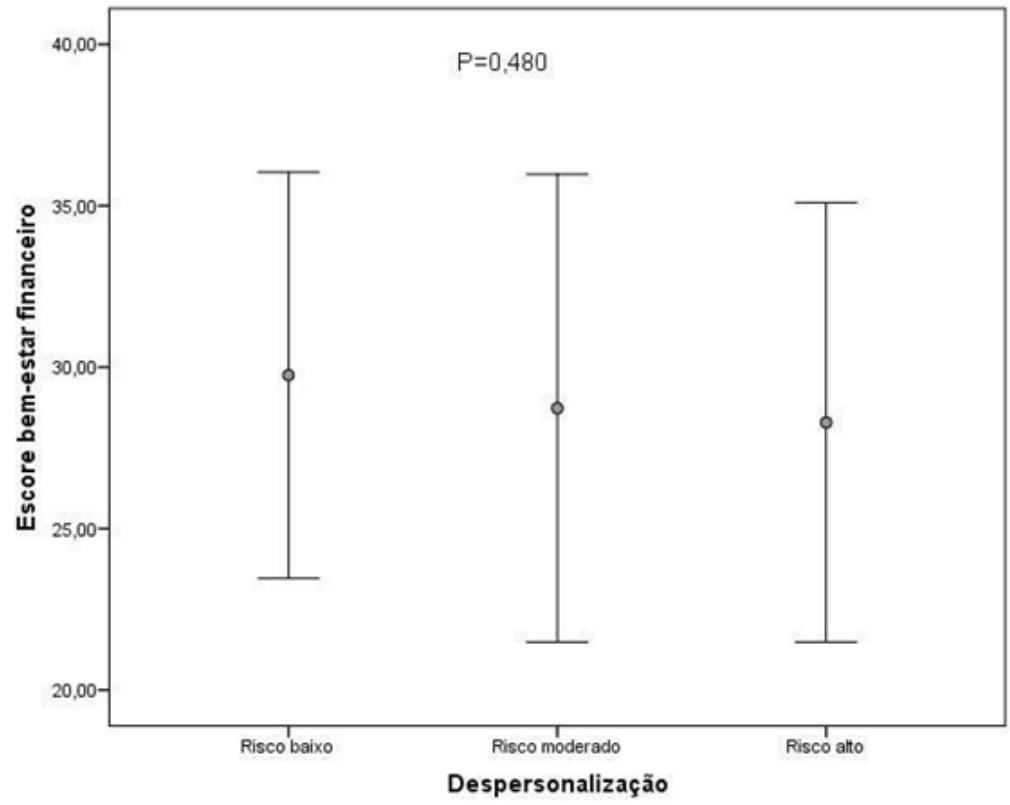

Figure 2 Relationship between Depersonalization and financial well-being. 


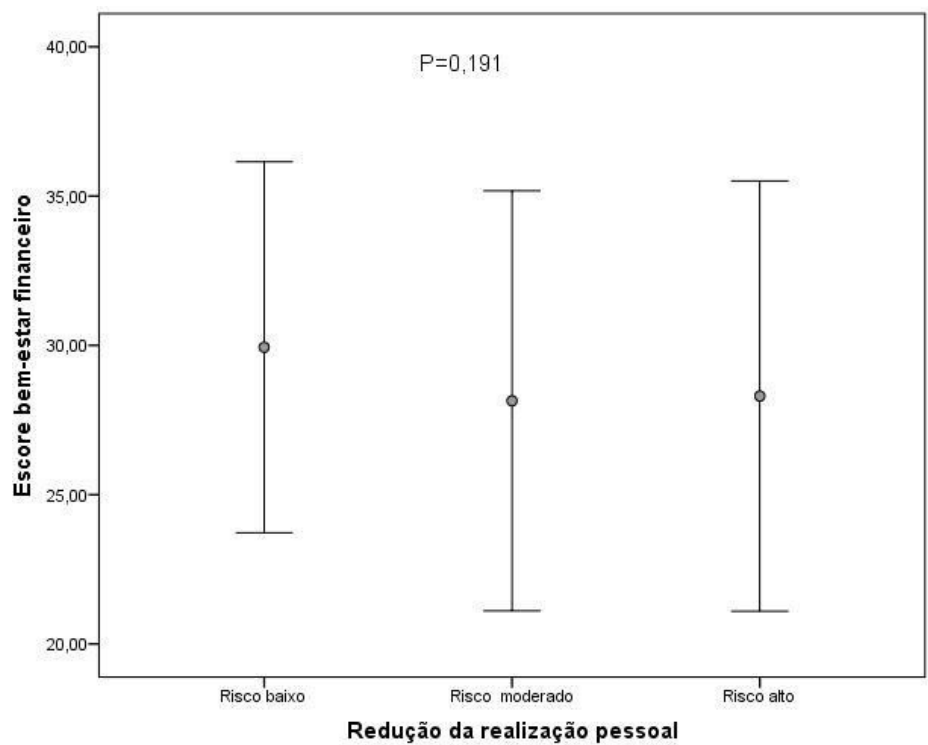

Figure 3 Relationship between Reduced personal fulfillment and financial well-being.

The other variables analyzed and their relationship with the risk of developing Burnout Syndrome symptoms are presented according to each dimension in Tables 3, 4 and 5. The variables "Age", "Years of work" and "Weekly workload" obtained statistical significance and demonstrated similar behavior for the three spheres. The high- risk group presents professionals with lower age, fewer years of experience, and higher weekly workload. In addition to these variables, the "Number of financial dependents" obtained statistical significance only for the sphere Reduction of personal accomplishment.

Table 3 Relationship between variables and emotional exhaustion

\begin{tabular}{|c|c|c|c|c|}
\hline Emotional Exhaustion & Low Risk & Moderate Risk & High Risk & $\mathbf{p}$ \\
\hline Financial well-being score* & $30,7 \pm 6,0^{a}$ & $29.5 \pm 6.8^{\mathrm{a}, \mathrm{b}}$ & $26,9 \pm 6,3^{b}$ & 0,001 \\
\hline Age in years* & $47,9 \pm 13,7^{\mathrm{a}}$ & $43,2 \pm 10,9^{b}$ & $39,8 \pm 8,8^{b}$ & $<0,001$ \\
\hline \multicolumn{5}{|l|}{$\operatorname{Sex}(\%)$} \\
\hline Male & 67,0 & 59,4 & 60,0 & \multirow[t]{2}{*}{0,511} \\
\hline Female & 33,0 & 40,6 & 40,0 & \\
\hline \multicolumn{5}{|l|}{ Marital status (\%) } \\
\hline Married or in a stable union & 75,4 & 79,4 & 69,1 & \multirow{2}{*}{0,758} \\
\hline Single & 18,4 & 14,3 & 23,6 & \\
\hline Divorced or widowed & 6,1 & 6,3 & 7,3 & \\
\hline \multicolumn{5}{|l|}{ Way of acting (\%) } \\
\hline Private Team & 58,3 & 76,6 & 60,0 & \\
\hline Autonomous & 21,7 & 9,4 & 10,9 & \\
\hline Resident & 9,6 & 9,4 & 18,2 & 0,072 \\
\hline $\begin{array}{l}\text { Contract with hospitals or } \\
\text { clinics }\end{array}$ & 7,0 & 1,6 & 3,6 & \\
\hline Civil Servant & 3,5 & 3,1 & 7,3 & \\
\hline Weekly workload* & $43,9 \pm 18,9^{a}$ & $46,8 \pm 19,8^{a}$ & $56,2 \pm 20,0^{b}$ & 0,001 \\
\hline Years in business\# & $20,0(6,0-30,0)^{\mathrm{a}}$ & $12.0(5.3-22.8)^{a, b}$ & $8,0(2,0-18,0)^{b}$ & $<0,00$ I \\
\hline Financial Dependents\# & $1,0(0,0-2,0)$ & $2,0(0,0-2,0)$ & $\mathrm{I}, 0(0,0-3,0)$ & 0,544 \\
\hline
\end{tabular}

*Variables with normal distribution described by mean \pm standard deviation and compared by ANOVAtest followed by Tukey; \#Variables with asymmetric distribution described by median (interquartile range: P25-P75) andcompared by Kruskal Wallis test $\%$ Categorical variables described by the percentage of $n$ and compared by the Chi-square test a,b different letters represent different categories statistically 
Table 4 Relationship of variables with depersonalization

\begin{tabular}{|c|c|c|c|c|}
\hline Depersonalization & Low risk & Moderate risk & High risk & $\mathbf{p}$ \\
\hline Financial well-being score* & $29,8 \pm 6,3$ & $28,7 \pm 7,2$ & $28,3 \pm 6,8$ & 0,480 \\
\hline Age in years* & $46,2 \pm 12,9^{a}$ & $39,6 \pm 8,3^{b}$ & $40.2 \pm 10.1^{a, b}$ & 0,004 \\
\hline \multicolumn{5}{|l|}{$\operatorname{Sex}(\%)$} \\
\hline Male & 61,7 & 63,6 & 76,2 & \multirow[t]{2}{*}{0,425} \\
\hline Female & 38,3 & 36,4 & 23,8 & \\
\hline \multicolumn{5}{|l|}{ Marital status (\%) } \\
\hline Married or in a stable union & 77,0 & 66,7 & 71,4 & \multirow{2}{*}{0,723} \\
\hline Single & 16,9 & 24,2 & 23,8 & \\
\hline Divorced or widowed & 6,2 & 9,1 & 4,8 & \\
\hline \multicolumn{5}{|l|}{ Way of acting (\%) } \\
\hline Private Team & 65,6 & 51,5 & 66,7 & \multirow{3}{*}{0,533} \\
\hline Autonomous & 16,1 & 18,2 & 9,5 & \\
\hline Resident & 9,4 & 18,2 & 19,0 & \\
\hline $\begin{array}{l}\text { Contract with hospitals or } \\
\text { clinics }\end{array}$ & 4,4 & 9,1 & 0,0 & \\
\hline Civil Servant & 4,4 & 3,0 & 4,8 & \\
\hline Weekly workload* & $46,0 \pm 18,8^{a}$ & $55,9 \pm 22,9^{b}$ & $48.4 \pm 22.0^{\mathrm{a}, \mathrm{b}}$ & 0,032 \\
\hline Years in business\# & $18,0(5,0-28,0)^{\mathrm{a}}$ & $8,0(3,0-18,0)^{b}$ & $8,0(2,5-18,5)^{b}$ & 0,003 \\
\hline Financial Dependents\# & $2,0(0,0-2,0)$ & $1,0(0,0-2,0)$ & $2,0(0,0-2,0)$ & 0,246 \\
\hline
\end{tabular}

*Variables with normal distribution described by mean \pm standard deviation and compared by ANOVAtest followed by Tukey; \# Variables with asymmetric distribution described by median (interquartile range: P25-P75) andcompared by Kruskal Wallis test $\%$ Categorical variables described by the percentage of $n$ and compared by the Chi-square test

a,b different letters represent different categories statistically

Table 5 Relationship of variables with personal accomplishment

\begin{tabular}{|c|c|c|c|c|}
\hline Personal fulfillment & Low risk & Moderate risk & High risk & $\mathbf{p}$ \\
\hline Financial well-being score* & $29,9 \pm 6,2$ & $28, I \pm 7,0$ & $28,3 \pm 7,2$ & 0,191 \\
\hline Age in years* & $46,2 \pm 12,7^{\mathrm{a}}$ & $41.8 \pm 10.4^{a, b}$ & $37,7 \pm 10,3^{b}$ & 0,003 \\
\hline \multicolumn{5}{|l|}{ Sex (\%) } \\
\hline Male & 61,6 & 71,4 & 60,0 & \multirow[t]{2}{*}{0,474} \\
\hline Female & 38,4 & 28,6 & 40,0 & \\
\hline \multicolumn{5}{|l|}{ Marital status (\%) } \\
\hline Married or in a stable union & 76,6 & 73,2 & 65,0 & \multirow{2}{*}{0,767} \\
\hline Single & 17,0 & 22,0 & 25,0 & \\
\hline Divorced or widowed & 6,4 & 4,9 & 10,0 & \\
\hline \multicolumn{5}{|l|}{ Way of acting (\%) } \\
\hline Private Team & 66,3 & 61,9 & 45,0 & \\
\hline Autonomous & 16,3 & 14,3 & 15,0 & \\
\hline Resident & 9,3 & 14,3 & 25,0 & 0,323 \\
\hline $\begin{array}{l}\text { Contract with hospitals or } \\
\text { clinics }\end{array}$ & 5,2 & 2,4 & 5,0 & \\
\hline Civil Servant & 2,9 & 7,1 & 10,0 & \\
\hline
\end{tabular}

Citation: Reimann DES, Bagatini DA. Relationship between financial well-being and Burnout Syndrome in anesthesiologists.J Anesth Crit Care Open Access. 2021;13(4):134-140. DOI: I0.15406/jaccoa.202I.13.00484 
Table Continued...

\begin{tabular}{lllll}
\hline Personal fulfillment & Low risk & Moderate risk & High risk & P \\
\hline Weekly workload* & $45,7 \pm 19,8 \mathrm{a}$ & $53,9 \pm 19,0 \mathrm{~b}$ & $5 \mathrm{I} .4 \pm 20.3 \mathrm{a}, \mathrm{b}$ & 0,039 \\
Years in business\# & $18,0(6,0-25,8)^{\mathrm{a}}$ & $10.0(3.0-20.0)^{\mathrm{a}, \mathrm{b}}$ & $6,0(2,0-12,5)^{\mathrm{b}}$ & $0,00 \mathrm{I}$ \\
Financial Dependents\# & $2,0(0,0-2,0)^{\mathrm{a}}$ & $1.0(0.0-2.0)^{\mathrm{a}, \mathrm{b}}$ & $0,5(0,0-1,8)^{\mathrm{b}}$ & 0,028 \\
\hline
\end{tabular}

*Variables with normal distribution described by mean \pm standard deviation and compared by ANOVAtest followed by Tukey;

\#Variables with asymmetric distribution described by median (interquartile range:P25-P75) andcompared by KruskalWallis test

$\%$ Categorical variables described by the percentage of $\mathrm{n}$ and compared by the Chi-square test

a,b different letters represent different categories statistically

\section{Discussion}

If we consider one of the three severely affected dimensions as a criterion to have Burnout Syndrome, we found a prevalence of $28.6 \%$ in our sample. As for the correlation between the syndrome and financial well-being, we found a statistically significant relationship between high risk for emotional exhaustion and a lower score in the financial well-being score $(30.7 \pm 6.0$ in the low risk group; $26.9 \pm 6.3$ in the high risk group). After adjusting for age and weekly workload, this result remained statistically significant. Regarding the other dimensions of Burnout, no statisticalsignificance was reached.

The author of the financial questionnaire used the sum of all answers as a parameter, without defining a cut-off point to classify the participants into different categories. Thus, we cannot classify financial well-being as bad or good, only quantify its score and compare it in a linear fashion. Despite this difference of only 3.8 points, it is important to emphasize the tendency of points to decrease concomitantly with the increased risk of Emotional Exhaustion as shown in Figure 1.It should be noted the importance of the statistical significance after adjusting for age and weekly workload, because they are variables that also obtained statistical significance in their comparisons.

The prevalence of the syndrome shows great variability in the literature, andthe rate of our sample diverges from other studies. A study conducted by the Federal Council of Medicine in 2007 found a prevalence of $57 \%$ in physicians. ${ }^{3}$ Data from 2012 in the United States presented $45 \%$ of the physicians with high scores in the dimensions Emotional Exhaustion and Depersonalization, and the rate of anesthesiologists was slightly higher than that of physicians in general. ${ }^{22}$ A survey carried out by the Medscape website and published in December of 2020 demonstrated a prevalence of $22 \%$ in professionals between 25 and 39 years of age. ${ }^{(23)}$ Studies with anesthesiologists in Brazil also found variable data. Data from Distrito Federal showed $10.4 \%$ of the interviewees with the syndrome, ${ }^{24}$ while another carried out at Faculdade de Medicina do ABC (Santo André) showed that $79.4 \%$ had medium to high risk to develop it. ${ }^{5}$

Emotional Exhaustion is considered the first stage of the syndrome and playsa central role in the Burnout ${ }^{2}$ experience, being directly related to high levels of demand at work. ${ }^{6,25}$ In our sample, Emotional Exhaustion presented higher prevalence of high risk in relation to the other dimensions (Emotional Exhaustion: 23.5\%; Depersonalization: 9.0\%; Reduced personal achievement: $8.5 \%$ ). These results are divergent from other Brazilian studies that found a higher proportion ofhigh risk in the dimension Depersonalization. Data from the Distrito Federal indicate prevalence of Emotional Exhaustion 23.1\%; Depersonalization 28.3\% and Reduced personal accomplishment $13.4 \%{ }^{24}$ and from ABC Santo André of Emotional Exhaustion $30.5 \%$; Depersonalization $33.9 \%$ and Reduced personal accomplishment $25.4 \%{ }^{5}$
The mean score of the financial well-being score was $29.5 \pm 6.5$, while the original work found a mean of $24.8 \pm 5.9 .{ }^{26}$ It should be noted that their sample is composed of students, while that of our study comprises only Anesthesiologists and Anesthesiology Residents. In the 2020 survey on the Medscape website, professionals were asked what would help reduce Burnout in their opinion. Increased pay to avoid financial stress was the most common response $(53 \%)$. The second most frequent response was more paid time off $(37 \%) .{ }^{23}$ Still, several studies show the negative impact of debts on mental health, with increased risk of depression and anxiety symptoms. ${ }^{11-14}$ Our results also follow this trend. Thus, we observe the clear importance of financial well-being on the mental health of a professional. Several risk factors for Burnout are related to personal life and work environment. One should consider that low financial well-being has a correlation with the syndrome and, possibly, is also a risk factor for its development.

Some characteristics also showed a statistically significant correlation with higher risk in the three dimensions: age, weekly workload and years of experienceAll showed the same trend among the dimensions: the younger the age, the fewerthe number of years of work, and the higher the weekly workload, the higher the risk. Similar data were found in the 2020 Medscape survey, which shows a higher prevalence of the syndrome in the younger age group,,$^{23}$ and in the study fromDistrito Federal which shows a lower mean age in professionals with the syndrome. ${ }^{24}$ Other variables studied, such as gender, marital status and type of work were not statistically significant, even though there was a predominance of males $(63.2 \%)$, married $(56.8 \%)$ and working in private anesthesiology teams $(63.7 \%)$.

The response rate to the study $(17.2 \%)$ is similar to other national studies involving the prevalence of Burnout Syndrome in physicians..$^{27,28}$ However, it is considered low and is one of the limitations of our work. In addition, it is important to stress that the results do not indicate a causal relationship, only a correlation between the variables. We can suppose that financial problems add to other risk factors to which an anesthesiologist is exposed, such as excessive workload and few leisure activities, leading the professional to develop Burnout Syndrome. Another hypothesis is that professionals with symptoms of burnout pay less attention to their personal finances and present lower financial well-being. Despite the difficulty in establishing a causal relationship, it is known that the variables mentioned above are correlated. This may help identify individuals who present a risk of developing the syndrome and thus enable its prevention.

As a contribution of this study, we could sum the characteristics that presented statistically significant correlation with high risk for Burnout Syndrome and outline a profile of characteristics of the anesthesiologist most susceptible to develop it. This professional presents lower financial well-being, younger age, shorter time of 
practice, and longer workload. It is worth mentioning that financial well-being is not related to higher or lower nominal values, it only reflects the feeling and subjective sensation in relation to the subject. Thus, this study makes it possible to reflect onthe healthy balance between workload and mental health.

\section{Conclusion}

This study found a statistically significant relationship between a lower financial well-being score and a higher risk of emotional exhaustion (one of the three dimensions of Burnout). The prevalence of the syndrome in the sample was $28.6 \%$. Other variables analyzed such as age, time of work and workload also showed a statistically significant correlation with higher risk.

Works correlating the variables Burnout and financial well-being were notfound in the researched platforms. The data presented in this work are of great importance to help understand which behaviors may be behind the development of the syndrome. Given the negative consequences of the syndrome and dysfunctional financial behavior, more studies are needed to elucidate the complex relationship between these important variables.

\section{Acknowledgments}

None.

\section{Conflicts of interest}

None.

\section{References}

1. Maslach C, Jackson SE. The measurement of experienced burnout. $J$ Organ Behav. 1981;2(2):99-113.

2. Grunfeld E, Whelan TJ, Zitzelsberger L, et al.Cancer care workers in Ontario: Prevalence of burnout, job stress and job satisfaction. CMAJ. 2000;163(2):166-169.

3. Andrade E de O, Barbosa GA, Brandão MC, et al. Conselho FEDERAL DE MEDICINA A SAÚDE DOS MÉDICOS DO BRASIL Brasília- DF [Internet]. 2007. 149 p. Neto GFD, Brazilian Society of Anesthesiology. Occupational Well-being in Anesthesiologists [Internet]. 2017. 1-289 p.

4. Serralheiro FC, Braga ALF, Garcia MLB, et al. Prevalence of Burnout syndrome in anesthesiologists from a Higher Education Institution in Medicine. Arq Bras Ciências da Saúde. 2011;36(3):140-143.

5. Cordes CL, Dougherty TW. a Review and an Integration of Research on Job Burnout. Acad Manag Rev. 1993;18(4):621-656.

6. Peckham C. Physician Burnout: It Just Keeps Getting Worse [Internet]. 2015.

7. Teixeira VB, Teixeira PJR, Rocha FL. Mental health in anesthesiology and burnout syndrome. Rev Médica Minas Gerais. 2016;26(Supl 1):15-21.

8. Calumbi RA, Amorim JA, Maciel CMC, Damázio Filho O, Teles AJF. Avaliação da qualidade de vida dos anestesiologistas da cidade do Recife. Rev Bras Anestesiol. 2010;60(1):42-51.
9. Narges D, Laily HP. Determinants of financial wellness among Malaysia workers. African J Bus Manag. 2011;5(24):10092-10100.

10. Bridges S, Disney R. Debt and depression. J Health Econ [Internet]. 2010;29(3):388-403.

11. Drentea P, Reynolds JR. Neither a borrower nor a lender be: The relative importance of debt and SES for mental health among older adults. J Aging Health.2012;24(4):673-695.

12. Turunen E, Hiilamo H. Health effects of indebtedness: A systematic review.BMC Public Health. 2014;14(1).

13. Bemel JE, Brower C, Chischillie A, et al. The Impact of College Student Financial Health on Other Dimensions of Health. Am J Heal Promot. 2016;30(4):224-230.

14. Richardson T, Elliott P, Roberts R. The relationship between personal unsecured debt and mental and physical health: A systematic review and meta-analysis. Clin Psychol Rev. 2013;33(8):1148-1162.

15. Clayton M, Liñares-Zegarra J, Wilson JOS. Does debt affect health? Cross country evidence on the debt-health nexus. Soc Sci Med. 2015;130:51-58.

16. French D, McKillop D. The impact of debt and financial stress on health in Northern Irish households. J Eur Soc Policy. 2017;27(5):458-473.

17. Demirguc-Kunt A, Klapper L, Singer D, et al. The Global Findex Database 2017: Measuring Financial Inclusion and the Fintech Revolution. The Global Findex Database 2017: Measuring Financial Inclusion and the Fintech Revolution. 2018.

18. Weber A, Jaekel-Reinhard A. Burnout Syndrome: A Disease of Modern Societies? Occup Med (Chic Ill). 2000;50(7):512-517.

19. Jackson SH. The role of stress in anaesthetists' health and well-being. Acta Anaesthesiol Scand. 1999;43(6):583-602.

20. Tamayo MR. Relationship between burnout syndrome and organizational values in the nursing staff of two public hospitals. Universidade de Brasília, DF; 1997.

21. D Shanafelt T, Boone S, Tan L, et al. Burnout and Satisfaction With Work-Life Balance Among US Physicians Relative to the General US Population. Arch Intern Med. 2012;172(18):1377-1385.

22. Schwartz L. Lifestyle and physician burnout in Brazil 2020 [Internet]. 2020.

23. Magalhães E, Oliveira ÁCM de S, Govêia CS, et al. Prevalence of burnout syndrome among anesthesiologists in Distrito Federal. Brazilian J Anesthesiol [Internet]. 2015;65(2):104-110.

24. Felton JS. Burnout as a clinical entity - its importance in health care workers. 2000;48(4):237-250.

25. Norvilitis JM, Szablicki PB, Wilson SD. Factors influencing levels of credit-card debt in college students. J Appl Soc Psychol. 2003;33(5):935-947.

26. De Melo Tucunduva LTC, Garcia AP, Boas Prudente FV, et al. The professional fatigue syndrome in Brazilian cancer physicians. Rev Assoc Med Bras. 2006;52(2):108-112.

27. Glasberg J, Horiuti L, Novais MAB, et al. Prevalence of the burnout syndrome among Brazilian medical oncologists. RevAssoc Med Bras. 2007;53(1):85-89. 